http://jmscr.igmpublication.org/home/

ISSN (e)-2347-176x ISSN (p) 2455-0450

crossref DOI: https://dx.doi.org/10.18535/jmscr/v7i10.118

Journal Of Medical Science And Clinical Research

IGM Publication

An Official Publication of IGM Publication

\title{
A Study of Serum IgE levels and Absolute Eosinophil Count in COPD
}

\author{
Authors
}

\author{
K.A. Vivek, N. Vijayakumar, R. Umarani
}

\begin{abstract}
Aims and Objectives: To study the prevalence of elevated serum total IgE levels and Absolute Eosinophil Count in Chronic Obstructive Pulmonary Disease (COPD) patients and its relation with GOLD staging. Methods: This study is a cross sectional study conducted on 50 inpatients presented with symptoms of COPD in the department of medicine in RMMCH during the period from September 2017 to October 2019. The studied group was divided according to the GOLD staging and compared in demographic data, clinical data, spirometric data, chest x-ray findings, serum total IgE levels and Absolute Eosinophil Count $(A E C)$.
\end{abstract}

Results: Out of the 50 patients enrolled in our study $84 \%(n=42)$ of patients were found to have elevated serum IgE levels, and 58\% (n=29) of patients were found to have elevated AEC levels. On comparing the serum IgE levels and Absolute Eosinophil Count with GOLD staging, there is a strong positive correlation between serum IgE, Absolute Eosinophil Count and GOLD staging, as the GOLD staging increases, the $I g E$ and $A E C$ also increases in a direct and linear fashion in our study subjects.

Conclusion: There was a high prevalence of elevated serum IgE levels and AEC in COPD patients. The mean serum total IgE levels and AEC increases as the GOLD stage increases.

\section{Introduction}

COPD is a heterogeneous disease and can be classified into different phenotypes. COPD is a disorder of progressive airflow limitation caused by chronic inflammation of the airways and lung parenchyma and associated with symptoms such as cough, sputum production, and dyspnea. Smoking is the primary risk factor for the development of COPD. Worldwide, it ranks as the fourth leading cause of death, alongside HIV/AIDS. By the year 2020, COPD is predicted to become the third leading cause of death worldwide (exceeded only by heart disease and stroke).

Exacerbation of asthma often have identifiable triggers such as allergens, cold air or exercise, but exacerbation in COPD patients are usually caused by infections. Both of these disease is considered to be at the opposite ends of the spectrum of airway disease, where bronchial asthma was thought to be highly responsive to treatment and reversible, COPD was characterized by fixed airway narrowing and unresponsive to the treatment. The currently accepted definitions still emphasize these features, but there may be a significant overlap between the two diseases. Today it is recognized that these two conditions are distinct but their clinical features overlap and is often difficult to differentiate, clinically diagnose ad classify the two disorders. The Dutch Hypothesis suggests that Asthma and COPD may be considered as different expressions of one disease entity. The purpose of this study is to evaluate the levels of total serum IgE levels and 
AEC in COPD patients and its relation to pulmonary function.

\section{Aims and Objectives}

1. To study the prevalence of elevated serum total IgE levels and AEC in Chronic Obstructive Pulmonary Disease (COPD)

2. To study the correlation between serum total IgE levels, AEC and GOLD staging.

\section{Inclusion Criteria}

1. Age more than 40 years,

2. Chronic cough, expectoration and/or wheeze for at least three months in a year for two successive years,

3. Currently smoking or having a history of smoking (smoking index P10 pack-year), and/evidence of irreversible obstructive impairment on spirometry

\section{Exclusion Criteria}

1. Receiving systemic steroid therapy in the preceding 4 weeks,

2. Receiving any other immunosuppressive therapy,

3. With active pulmonary tuberculosis, interstitial lung disease, and severe heart failure,

4. Not adherent to medication

5. Uncontrolled diabetes mellitus, thyroid disorders

6. No exacerbation in the last one month.

\section{Materials and Methods}

A cross sectional study was conducted on 50 patients admitted as inpatients presented with symptoms of COPD in the department of medicine in $\mathrm{RMMCH}$ during the period from September 2017 to October 2019. Patients were recruited based on the inclusion and exclusion criteria mentioned above and were classified as mild, moderate, severe and very severe according to GOLD criteria (FEV1/FVC $<0.70$ and post bronchodilator FEV1 > $80 \%$ of normal in mild COPD, FEV1 50-79 \% in moderate COPD, FEV1
$30-49 \%$ in severe COPD and FEV1 < 30\% in very severe COPD).

All patients were subjected to a complete history taking and physical examination. Under aseptic precaution 6-8 $\mathrm{ml}$ of blood was taken from the median cubital vein in all patients and was measured for complete blood count, renal function test, liver function test, absolute eosinophil count, peripheral smear and serum total IgE levels. Pulmonary function test, sputum for AFB and, stool analysis and chest $\mathrm{x}$-rays were also done in all patients.

These investigations were done to rule out diseases like active tuberculosis, parasitic infection, eosinophilic pneumonias, malignancy, chronic eosinophilic leukemia. Clinical findings, spirometry and radiology helped in ruling out asthma.

A volunteer written consent was taken from all the patients before enrolling to the study and the study was approved by Institutional Human Ethical Committee. All statistical analysis was performed using a statistical software package (Statistical Package for Social Sciences, SPSS 16.0). Comparisons of continuous data between two groups were performed by $\mathrm{T}$ - test and MannWhitney $U$ test. Comparisons of continuous data among four groups were performed by an ANOVA test and Kruskal Wallis test. Spearman correlation were used for correlation analysis. $\mathrm{P}$ value less than 0.05 were considered as statistically significant.

\section{Results and Analysis}

During the study period we studied 50 patients with COPD (40 males (80\%) and 10 females $(20 \%))$, and 6 patients (12\%) had mild COPD, 19 patients (38\%) had moderate COPD, 21 patients (42\%) had severe COPD, and 4 patients (8\%) had very severe COPD, disease classification was established according to GOLD criteria

All group of patients were age and sex matched without any significant difference. There was a significant difference between studied groups regarding clinal indices (Duration of illness, 
dyspnea, wheeze, smoking index). Serum total IgE levels and AEC showed a direct and linear relationship with GOLD staging, as the GOLD staging increases the serum IgE levels and AEC also increases. Chest radiograph showed emphysema in 37 patients (74\%) and chronic bronchitis in 13 patients (26\%) (see Table 1)
Table 2 shows correlation coefficient analysis of serum total IgE, AEC and FEV1 levels (GOLD Staging). There was a strong negative correlation between AEC and FEV1 levels, as the FEV1 decreases the AEC level increases. Similarly there was a strong negative correlation between serum IgE levels and FEV1 levels, as the FEV1 decreases the serum IgE level increases.

Table 1: Patients Characteristics

\begin{tabular}{|c|c|c|c|c|c|c|}
\hline \multicolumn{2}{|c|}{ Variables } & $\underset{(\mathbf{n}=\mathbf{6})}{\text { STAGE I }}$ & $\underset{\substack{\text { II } \\
(n=19)}}{\text { STAGE }}$ & $\begin{array}{c}\text { STAGE } \\
\text { III } \\
(\mathbf{n}=21)\end{array}$ & $\begin{array}{c}\text { STAGE } \\
\text { IV } \\
(\mathbf{n}=4)\end{array}$ & p-value \\
\hline \multicolumn{2}{|c|}{ Age (mean) } & 65.3 & 61.5 & 64.5 & 59.7 & $>0.05$ \\
\hline \multirow{2}{*}{ Sex } & Male & 5 & 16 & 17 & 2 & $>0.05$ \\
\hline & Female & 1 & 3 & 3 & 2 & $>0.05$ \\
\hline \multicolumn{2}{|c|}{ Dyspnea } & 4 & 14 & 18 & 4 & $<0.001$ \\
\hline \multicolumn{2}{|c|}{ Wheeze } & 3 & 16 & 18 & 4 & $<0.001$ \\
\hline \multicolumn{2}{|c|}{ Pack years (mean) } & 14.5 & 15.3 & 16.1 & 19.2 & $<0.001$ \\
\hline \multicolumn{2}{|c|}{$\begin{array}{l}\text { Duration of illness } \\
\text { (mean years) }\end{array}$} & 2.6 & 3.9 & 4.0 & 8.5 & \multirow{4}{*}{$<0.05$} \\
\hline \multirow{3}{*}{\multicolumn{2}{|c|}{$\begin{array}{l}<5 \text { years } \\
6-10 \text { years } \\
>10 \text { years }\end{array}$}} & 5 & 16 & 18 & 0 & \\
\hline & & 1 & 2 & 2 & 3 & \\
\hline & & 0 & 1 & 1 & 1 & \\
\hline \multicolumn{2}{|c|}{ FEV1 (mean) } & 80.4 & 59.5 & 36.6 & 27.6 & $<0.001$ \\
\hline \multicolumn{2}{|c|}{ Serum IgE levels (mean) } & 505.5 & 858.6 & 1291.3 & 2278.5 & $<0.001$ \\
\hline \multicolumn{2}{|c|}{ AEC (mean) } & 287.6 & 398.3 & 545.5 & 1341.5 & $<0.001$ \\
\hline \multirow{2}{*}{ Radiology } & Ch.bronchitis & 2 & 5 & 5 & 1 & \multirow{2}{*}{$<0.01$} \\
\hline & Emphysema & 4 & 14 & 16 & 3 & \\
\hline
\end{tabular}

Table 2: Correlation Statistics

\begin{tabular}{|l|c|c|}
\hline \multirow{2}{*}{ Parameters } & \multicolumn{2}{|c|}{ FEV1 } \\
\cline { 2 - 3 } & R & P VALUE \\
\hline AEC & -0.52 & $<0.01$ \\
\hline Serum IgE & -0.62 & $<0.01$ \\
\hline
\end{tabular}

\section{Discussion}

Our study showed that increase in serum total IgE is a sensitive marker for allergy, COPD patients with higher smoking index, longer duration of illness, more severe lung function impairment, also there was a strong association of serum total IgE levels and AEC.

Previous studies have demonstrated that allergy was associated with decreased lung function in general population, smokers and patients with mild COPD. The increase in serum total IgE can also result from allergic inflammation due to some other cause (allergic inflammation of some other parts of the host), and maybe even associated with genetic factors and smoking.
In our study, the increased levels of serum $\operatorname{IgE}$ and AEC was considered to mostly reflect the allergic inflammation of the airways. The prevalence of serum IgE levels and AEC were much lower in patients with early stages of COPD compared to late stages. Also there were no allergic inflammation of other systems such as skin was noted and parasitic infection was excluded in our patients according to history taking, physical examination and other accessory examinations.

The result showed that the prevalence of elevated serum total $\operatorname{IgE}$ was $84 \%$ and the prevalence of elevated AEC was 58\% respectively, implying that even among COPD patients without obvious atopy hypersensitive inflammation of the lower 
airway may exist, probably representing the real proportion of the allergic phenotypes in patients with COPD

The results also showed that the COPD patients with elevated serum total IgE levels and AEC suffered a longer duration of illness, had earlier onset of dyspnea, higher smoking index and had a poor pulmonary function. This suggest that the elevated serum IgE levels and AEC might to some degree have an effect on the progression of COPD or could be a useful marker to reflect the severity of the disease.

\section{Limitations}

There were several limitations to the study. We had a small sample size. The causative organisms were not found as the culture sensitivity was not done. The diagnosis of fungal causes like aspergilloma was not done. We did not analyze the difference in long term drug therapy particularly corticosteroids in these patients. The time interval between the exacerbations was analysed.

\section{Conclusion}

There was a high prevalence of elevated serum total IgE levels and AEC in COPD patients without obvious atopy. Serum IgE levels and AEC were found to be associated with symptoms such as breathlessness and impaired pulmonary function. The elevated serum IgE levels and AEC could be an useful marker to reflect the severity of the disease and disease progression. Further studies may also be needed to examine the potential role of allergy in disease expression and progression of disease.

\section{References}

1. ICMR-MRC Workshop. Building Indo-UK Collaboration in chronic diseases. 2009:16.

2. Salvi S, Agarwal A. India needs a national COPD prevention and Control program. J Assoc Physicians India. 2012;60(Suppl):5-7.
3. Lopez AD, Shibuya K, Rao C, Mathers CD, Hansell AL, Held LS, et al. Chronic obstructive airway disease: Current burden and future projections. Eur Resp J. 2006;27:397-412.

4. Vedel-Krogh S1,2,3, Nielsen SF1,2,3, Lange P2,4,5, Vestbo J6, Nordestgaard BG1,2,3. Blood Eosinophils and Exacerbations in Chronic Obstructive Pulmonary Disease. The Copenhagen General Population Study.

5. Netsanet ANegewo1, Vanessa M McDonald1-4 et al, Peripheral blood eosinophils: a surrogate marker for airway eosinophilia in stable COPD, International journal of COPD.

6. Dave Singh, Umme Kolsum, Chris E et al. Eosinophilic inflammation in COPD: prevalence and clinical characteristics, European Respiratory Journal 2014.

7. Vedel-Krogh S1,2,3, Nielsen SF1,2,3, Lange P2,4,5, Vestbo J6, Nordestgaard BG1,2,3. Blood Eosinophils and Exacerbations in Chronic Obstructive Pulmonary Disease. The Copenhagen General Population Study.

8. Umme Kolsum, 1 Gavin C. Donaldson, 2 Richa Singh,2 Bethan L. Barker, Blood and sputum eosinophils in COPD; relationship with bacterial load, ReRespir Res. 2017.

9. Virender P. Singh Rathod, Prem Kapoor, K. K. Pillai, and Razia Khanam, Assessment of asthma and chronic obstructive pulmonary disorder in relation to reversibility, $\operatorname{IgE}$, eosinophil, and neutrophil count in a University Teaching Hospital in South Delhi, India.

10. Singh B1, Arora S, Khanna V. Association of severity of COPD with IgE and interleukin-1 beta. Monaldi Arch Chest Dis. 2010 Jun;73(2):86-7. 